\title{
Effects of a swallowing and oral care intervention for patients following endotracheal extubation: a pre- and post- intervention study
}

Chung-Pei Wu' ${ }^{1}$, Yu-Juan $\mathrm{Xu}^{1}$, Tyng-Guey Wang ${ }^{2}$, Shih-Chi Ku ${ }^{3 *}$, Ding-Cheng Chan ${ }^{3,4,5}$, Jang-Jaer Lee ${ }^{6}$, Yu-Chung Wei ${ }^{7}$, Tzu-Yu Hsiao ${ }^{8}$ and Cheryl Chia-Hui Chen ${ }^{1 *}$

\begin{abstract}
Background: For patients who survive a critical illness and have their oral endotracheal tube removed, dysphagia is highly prevalent, and without intervention, it may persist far beyond hospital discharge. This pre- and postintervention study with historical controls tested the effects of a swallowing and oral care (SOC) intervention on patients' time to resume oral intake and salivary flow following endotracheal extubation.

Methods: The sample comprised intensive care unit patients ( $\geq 50$ years) successfully extubated after $\geq 48 \mathrm{~h}$ endotracheal intubation. Participants who received usual care (controls, $n=117$ ) were recruited before 2015, and those who received usual care plus the intervention $(n=54)$ were enrolled after 2015. After extubation, all participants were assessed by a blinded nurse for daily intake status (21 days) and whole-mouth unstimulated salivary flow $(2,7,14$ days). The intervention group received the nurse-administered SOC intervention, comprising toothbrushing/salivary gland massage, oral motor exercise, and safe-swallowing education daily for 14 days or until hospital discharge.
\end{abstract}

Results: The intervention group received $8.3 \pm 4.2$ days of SOC intervention, taking 15.4 min daily with no reported adverse event (coughing, wet voice, or decreased oxygen saturation) during and immediately after intervention. Participants who received the intervention were significantly more likely than controls to resume total oral intake after extubation (aHR 1.77, 95\% Cl 1.08-2.91). Stratified by age group, older participants ( $\geq 65$ years) in the SOC group were 2.47 -fold more likely than their younger counterparts to resume total oral intake (aHR $2.47,95 \% \mathrm{Cl}$ 1.31-4.67). The SOC group also had significantly higher salivary flows 14 days following extubation $(\beta=0.67,95 \% \mathrm{Cl}$ 0.29-1.06).

Conclusions: The nurse-administered SOC is safe and effective, with greater odds of patients' resuming total oral intake and increased salivary flows 14 days following endotracheal extubation. Age matters with SOC; it more effectively helped participants $\geq 65$ years old resume total oral intake postextubation.

Trial registration: NCT02334774, registered on January 08, 2015

Keywords: Endotracheal intubation, Dysphagia, Critical illness, Oral intake, Swallowing, Salivation

\footnotetext{
*Correspondence: scku1015@gmail.com; cherylchen@ntu.edu.tw

${ }^{3}$ Department of Internal Medicine, National Taiwan University Hospital and National Taiwan University College of Medicine, 1, Jen-Ai Rd., Section 1,

Taipei, Taiwan, Republic of China100

${ }^{1}$ Department of Nursing, National Taiwan University Hospital and National

Taiwan University College of Medicine, 1, Jen-Ai Rd., Section 1, Taipei,

Taiwan, Republic of China100

Full list of author information is available at the end of the article
}

(c) The Author(s). 2019 Open Access This article is distributed under the terms of the Creative Commons Attribution 4.0 International License (http://creativecommons.org/licenses/by/4.0/), which permits unrestricted use, distribution, and reproduction in any medium, provided you give appropriate credit to the original author(s) and the source, provide a link to the Creative Commons license, and indicate if changes were made. The Creative Commons Public Domain Dedication waiver (http://creativecommons.org/publicdomain/zero/1.0/) applies to the data made available in this article, unless otherwise stated. 


\section{Introduction}

For patients who survive a critical illness and have their oral endotracheal tube removed, dysphagia is highly prevalent and may persist far beyond hospital discharge [1]. Dysphagia after extubation affects up to $62 \%$ of intensive care unit (ICU) patients, especially for patients with prolonged $(\geq 48 \mathrm{~h}$ ) endotracheal intubation [2-6]. When dysphagia persists, resumption of oral intake is delayed, committing patients to feeding tube dependence $[6,7]$. In one large observational trial $(N=933)$ from Switzerland, dysphagia lasted until hospital discharge in $60.4 \%$ of adult ICU patients [2]. By 21 days after extubation, even for ICU patients without prior swallowing difficulties or known pathologies such as stroke or neuromuscular deficits, $15.5 \%$ had persistent dysphagia, could not resume oral intake, and were feeding tube dependent [6]. Indeed, without intervention, dysphagia symptoms were sustained far beyond hospital discharge; $23 \%$ of orally intubated ICU survivors had dysphagia persisting $>6$ months in a 5 -year longitudinal cohort study involving 13 ICUs at 4 US teaching hospitals [8].

Dysphagia after extubation negatively affects patient outcomes [1], leading to delayed resumption of oral intake $[6,7,9]$, poor life quality $[10,11]$, aspiration pneumonia $[4,8,10,12]$, longer ICU and hospital stays $[2,7]$, and increased 90-day mortality [2]. Evidence for dysphagia treatment, however, is limited [2] as few intervention studies have been designed to reduce dysphagia or the time needed to resume total oral intake after extubation [13].

Herein, we hypothesized that improving oral lubrication, oral sensation, and strength in the lips, tongue, jaw, and cheeks would reduce time to resume total oral intake and enhance salivary flow for patients who received prolonged endotracheal intubation and had no prior swallowing difficulties or known pathologies such as stroke or neuromuscular deficits.

Our previous studies of the sequelae of prolonged endotracheal intubation in this group of ICU patients $[6,14,15]$ revealed that reduced salivary flow [14], sensorimotor impairment of the tongue [15], poor lip seal [14], and restricted mouth opening (i.e., weakness of masticatory muscles to move the jaw) [14] were highly prevalent and may persist 14 days postextubation. As these sequelae contribute to dysphagia and delay oral intake after extubation $[1,16]$, we developed a nurse-administered, hospital-based (provided daily until hospital discharge, up to 14 days) swallowing and oral care (SOC) intervention comprising toothbrushing/salivary gland massage, oral motor exercise (i.e., range of motion $[\mathrm{ROM}]$ exercises for the lips, tongue, jaw, and cheeks), and safe-swallowing education.

We hypothesized that toothbrushing/salivary gland massage would enhance oral lubrication and oral sensation by mechanically stimulating oral sensory receptors and increasing salivary flow $[17,18]$. Oral motor exercise may alleviate patients' poor lip seal, reduced tongue strength, poor lingual agility, and restricted mouth opening by strengthening the lips, tongue, jaw, and cheeks $[19,20]$. While these two SOC protocols may facilitate recovery from the sequelae of prolonged endotracheal intubation, safe-swallowing education is important to include as a safety precaution to reduce the aspiration risk [16].

As primary endpoints, time to resume total oral intake (i.e., total oral diet with multiple consistencies, measured by $\geq$ level 6 on the functional oral intake scale [FOIS] and censored 21 days postextubation) and unstimulated whole-mouth salivary flow (measured by oral Schirmer's test 2,7 , and 14 days postextubation) were measured by blinded nurses. Swallowing was not evaluated instrumentally (i.e., videofluoroscopy or fiberoptic endoscopic evaluation), since these tests are invasive and our study was a pilot. Intervention feasibility was also evaluated based on patients' adherence to the SOC intervention, time spent providing SOC, and adverse events during the intervention.

\section{Methods}

Study design, settings, and participants

This pre- and post-intervention study with historical controls was conducted at a tertiary medical center in Taipei, Taiwan. From October 2012 to November 2015, participants were recruited from consecutive patients $(\geq$ 50 years) admitted to the medical center's six medical ICUs if they had received emergency oral endotracheal intubation for at least $48 \mathrm{~h}$. Patients were excluded if they (1) had a history of neuromuscular disease (e.g., stroke, Parkinsonism) or head and neck deformities, (2) had a preexisting swallowing difficulty, (3) received tracheostomy, (4) could not respond to questions/intervention, and (5) were absolutely quarantined (e.g., for open or infectious tuberculosis). All patients and/or their legal representative signed written informed consent. Participants recruited before 2015 served as controls; participants recruited after 2015 received usual care plus the SOC intervention and served as the intervention group (Fig. 1). As no prior studies on this topic were available, we could not estimate the intervention effect to determine the study size. Nonetheless, we included 54 participants in the SOC group. The study was approved by the Human Research Ethics Committee of the study hospital (201411079RIND) and registered at the Clinical Trials Registry (trial no: NCT02334774).

\section{Usual care}

Usual care consisted of standard hospital care provided by ICU physicians and nurses. Upon extubation, oral intake was withheld until participants demonstrated no signs of choking (i.e., coughing, drooling, wet voice, or decreased oxygen saturation) on a small amount of water, progressing 


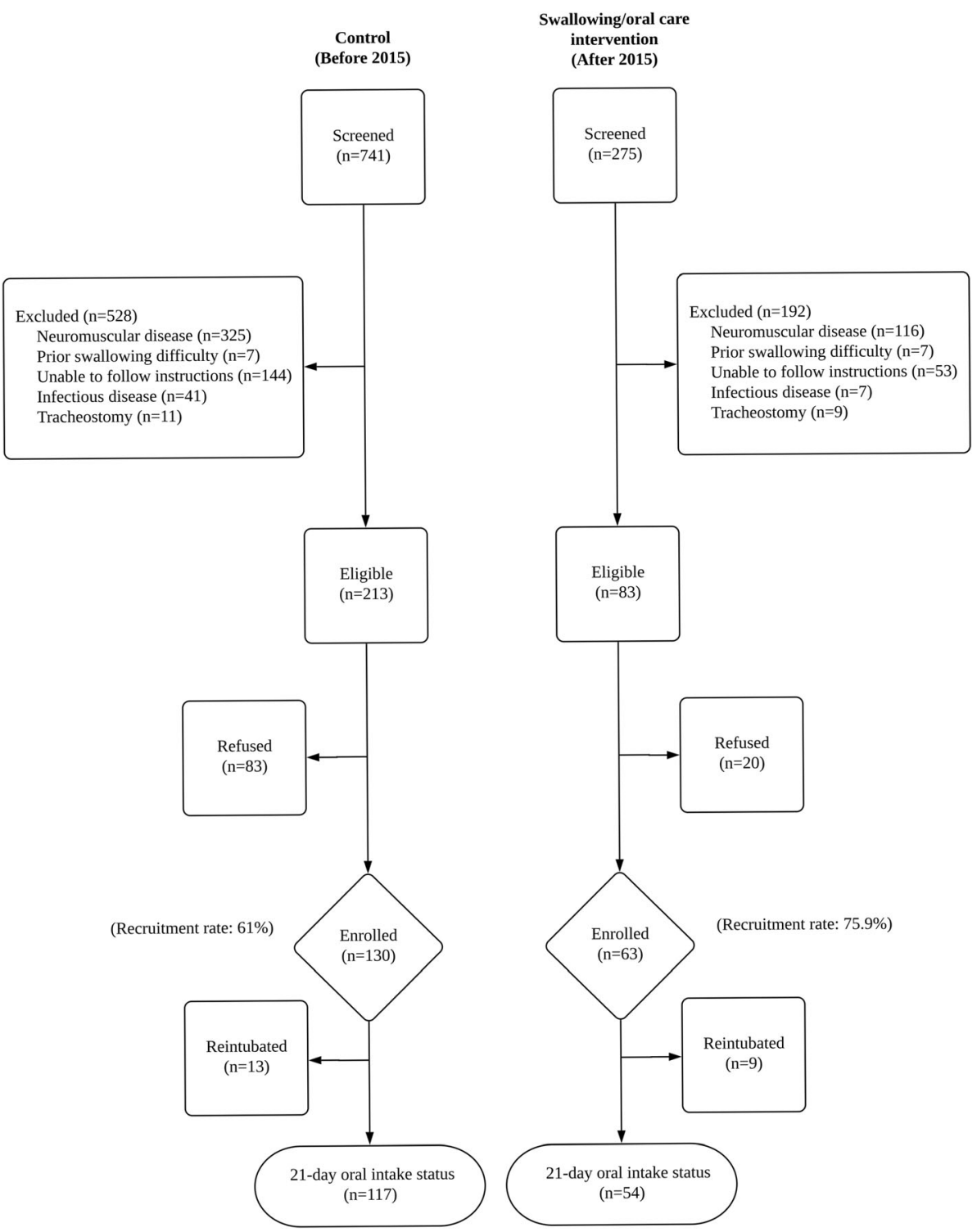

Fig. 1 Study flow diagram

to small and consistent amounts of food/liquid as tolerated. All participants were encouraged to take food/liquid orally and did so as tolerated. A speech therapist or dietician provided additional care only at the attending physician's request. Oral care was provided each shift by ICU nurses using oral swabs and rinsing with $2 \%$ chlorhexidine gluconate. Once patients were transferred to a general ward, oral care was considered a self-care process that was often assisted by family members.

\section{SOC intervention}

After intervention group participants were successfully extubated, the SOC intervention was administered on the next day (regardless of intake status) and daily until hospital discharge or 14 days postextubation (whichever occurred first). This intervention schedule was based on our observation that sequelae of endotracheal intubation may persist 14 days [14]. A trained SOC nurse, equipped with cheek retractor, dental suction tube, and tongue holder, provided the SOC intervention, i.e., toothbrushing/salivary gland massage, oral motor exercise, and safe-swallowing education (Additional file 1). Briefly, the SOC nurse brushed participants' oral cavity (teeth/gum, tongue, and palate) with a soft toothbrush using distilled water to remove the coated plaque, mechanically stimulate tissues, and rinse the oral cavity. The SOC nurse then moisturized participants' lips with Vaseline $e^{\circ}$ before placing fingers on participants' cheeks and gently massaging/pressing the surface overlying the parotid, sublingual, and submandibular salivary glands. Participants 
were then asked to purse the lips, move the tongue, open the mouth widely, and inflate the cheeks (each with 3,5 , or 10 repetitions/with or without resistance, depending on participants' tolerance) as one round of ROM exercises for the lips, tongue, jaw, and cheeks. Based on participants' intake status, brief safe-swallowing education was offered daily, explaining the signs and symptoms of unsafe swallowing and providing tips on sitting up to eat and modifying dietary texture and viscosity for patients and their family caregivers to reduce the aspiration risk. Before implementing the intervention, the SOC nurse was trained on-site for 2 months by experienced ICU nurses, a physician in rehabilitative medicine, and speech-language pathologists. Speech-language pathologists coached SOC nurse on identifying adverse events (such as wet voice) and gave practical tips on salivary gland massage and oral motor exercises of the lips, tongue, jaw, and cheeks in the ICU setting.

\section{Data collection and outcome measures}

Data on participant characteristics (age, gender, education [years], current smoker [yes/no], body mass index, admission diagnosis [respiratory failure, septic shock/ sepsis, heart disease, gastrointestinal bleeding, other], illness severity [Acute Physiological and Chronic Health Evaluation II (APACHE II) 0-24, 2 25]) were abstracted from medical records. Baseline data on oxygen supplementation (simple mask, non-rebreathing mask, bi-level positive airway pressure [yes/no]), intake level (FOIS level 1 , levels $2-3$, levels $4-7$ to indicate nothing by mouth, tube feeding with intake attempts, and oral intake), and salivary flow $(\mathrm{cm} / 5 \mathrm{~min})$ were evaluated by a research nurse at enrollment (before intervention).

All participants' daily intake status was evaluated for 21 days postextubation by a research nurse using the FOIS, a validated tool with established validity (81-98\%) and inter-rater reliabilities (0.86 to 0.91) [21]. FOIS scores range from levels 1 to 7 , with levels 1 through 3 indicating varying degrees of tube feeding and levels 4 through 7 indicating varying degrees of oral intake [21]. We considered FOIS level 6 (total oral diet with multiple consistencies, without special preparation but with specific food limitations) or 7 (total oral diet with no restriction) as "resume total oral intake." Unstimulated whole-mouth salivary flow was evaluated 2,7 , and 14 days postextubation using the oral Schirmer's test [22]. Considering response burden and time required for changes, the oral Schirmer's test, unlike the FOIS, was not measured daily. With participants sitting upright, research nurses held a standardized 1 -cm-wide by $17-\mathrm{cm}$ long Schirmer's tear test strip vertically, with the rounded end of the strip at the floor of their mouth. At the end of $5 \mathrm{~min}$, a wetting length in centimeter was recorded.
Feasibility of the SOC intervention was evaluated by time spent providing the SOC, patients' adherence to SOC components, and adverse events (i.e., coughing, wet voice, and decreased oxygen saturation) during and immediately after the intervention. Adherence was calculated as the percentage of the session that a participant completed compared to the maximal session that a participant could receive (maximum 14). Rates were documented separately for three SOC components (toothbrushing/salivary gland massage, oral motor exercise, and safe-swallowing education).

\section{Statistical analysis}

Participants' characteristics were analyzed by frequency (percentage) and mean (standard deviation) or median (interquartile range). Differences in characteristics by group were analyzed using the non-parametric MannWhitney $U$ test for continuous variables and Fisher's exact test for categorical variables.

Time to resume total oral intake for the SOC and control groups was plotted using the Kaplan-Meier survival curves. Considering age and baseline-cohort differences, participants' between-group clinical characteristics that differed $(P<0.1$, Table 1$)$ were entered as fixed covariates (non-time dependent) in Cox proportional hazard modeling and reported as adjusted hazard ratios (aHR). Salivary flows 2, 7, and 14 days following extubation were compared between the SOC and control groups by generalized estimating equations (GEE). An interaction term, "time by group," was used to indicate the betweengroup slope difference over time. Analyses were performed on a per-protocol approach, and no data were imputed. All analyses were performed using IBM SPSS Statistics (version 21, 2012, IBM Corporation). Significance was set at $P<0.05$.

\section{Results}

The SOC intervention was successfully implemented with differences in baseline characteristics noted between the SOC and control groups (Table 1). Namely, participants in the SOC group were older ( $71.4 \pm 10.8$ years) than controls (68.2 \pm 10.8 years, $P=0.39)$ and their body weight $(59.5 \pm$ $11.5 \mathrm{~kg})$ was lower than that of controls $(64.1 \pm 15.1 \mathrm{~kg}, P=$ $0.44)$. Within $48 \mathrm{~h}$ postextubation, a higher percentage of SOC participants received oxygen supplementation (70.4\%) than controls $(53.9 \%, P=0.05)$. Most importantly, baseline intake level was significantly worse for the SOC group (63.0\% were at FOIS level 1 [nothing by mouth]) than for the control group $(41.0 \%$ were at FOIS level $1 ; P<0.01)$. Conversely, only $5.6 \%$ of the SOC group vs. $30.8 \%$ of controls were at FOIS levels 4-7. Notably, no participant's intake was withheld due to gastrointestinal causes such as bleeding, although 11 participants were diagnosed at admission with gastrointestinal bleeding. As to salivary flow, the SOC group 
Table 1 Participant characteristics by group

\begin{tabular}{|c|c|c|c|}
\hline Characteristic & SOC $(n=54)$ & Control $(n=117)$ & $P$ value \\
\hline \multicolumn{4}{|l|}{ Demographic } \\
\hline Age, years, mean (SD) & $71.4(10.8)$ & $68.2(10.8)$ & $0.39^{a}$ \\
\hline Female gender, $n(\%)$ & $21(38.9)$ & $42(35.9)$ & $0.74^{\mathrm{b}}$ \\
\hline Education, years, mean (SD) & $9.4(5.3)$ & $9.9(4.8)$ & $0.94^{\mathrm{a}}$ \\
\hline Current smoker, $n(\%)$ & $9(17.0)$ & $30(25.9)$ & $0.24^{\mathrm{b}}$ \\
\hline Body weight, mean (SD) & $59.5(11.5)$ & $64.1(15.1)$ & $0.44^{\mathrm{a}}$ \\
\hline Body mass index, mean (SD) & $22.9(4.0)$ & $23.8(5.3)$ & $0.49^{\mathrm{a}}$ \\
\hline \multicolumn{4}{|l|}{ Clinical } \\
\hline Admission diagnosis, $n(\%)$ & & & $0.83^{b}$ \\
\hline Respiratory failure & $23(42.6)$ & $53(45.3)$ & \\
\hline Septic shock/sepsis & $14(25.9)$ & $28(23.9)$ & \\
\hline Heart disease & $12(22.2)$ & $18(15.4)$ & \\
\hline Gastrointestinal bleeding & $3(5.6)$ & $8(6.8)$ & \\
\hline Other & $2(3.7)$ & $10(8.5)$ & \\
\hline APACHE II score, $n(\%)$ & & & $0.13^{b}$ \\
\hline $0-24$ & $26(48.1)$ & $72(61.5)$ & \\
\hline$\geq 25$ & $28(51.9)$ & $45(38.5)$ & \\
\hline Intake level, $n(\%)$ & & & $<0.01^{\mathrm{b}}$ \\
\hline FOIS level 1 & $34(63.0)$ & $48(41.0)$ & \\
\hline FOIS levels 2-3 & $17(31.5)$ & $33(28.2)$ & \\
\hline FOIS levels 4-7 & $3(5.6)$ & $36(30.8)$ & \\
\hline Oxygen supplementation, $n$ (\%) & $38(70.4)$ & $62(53.9)$ & $0.05^{\mathrm{b}}$ \\
\hline Salivary flow, cm/5 min, median (IQR) & $3.0(3.0)$ & $3.9(2.0)$ & $0.03^{\mathrm{a}}$ \\
\hline
\end{tabular}

FOIS functional oral intake scale, SOC swallowing and oral care, APACHE Acute Physiological and Chronic Health Evaluation. FOIS Level 1, nothing by mouth; level 2 , tube dependent with minimal attempts of food or liquid; level 3, tube dependent with consistent oral intake of food or liquid; level 4, total oral diet of a single consistency; level 5, total oral diet with multiple consistencies, but requiring special preparation or compensations; level 6, total oral diet with multiple consistencies with special preparation, but with specific food limitations; level 7, total oral diet with no restrictions

${ }^{a}$ Mann-Whitney $U$ test

${ }^{\text {b} F i s h e r ' s ~ e x a c t ~ t e s t ~}$

also had a significantly lower baseline salivary flow $(3.0 \mathrm{~cm} / 5$ $\mathrm{min}$ ) than the controls $(3.9 \mathrm{~cm} / 5 \mathrm{~min}, P=0.03)$.

\section{Resume total oral intake}

Overall, $45.6 \%$ of participants did not resume total oral intake by 21 days postextubation, but the SOC group took less time to resume total oral intake ( $14 \pm 4.3$ days) than controls $(16 \pm 2.2$ days) although this difference did not reach significance. After adjusting for three baseline between-group differences (age, intake level, oxygen supplementation), the SOC group was significantly more likely to resume total oral intake (aHR 1.77, 95\% CI 1.08-2.91) than controls (Table 2). Stratified by age group, older participants ( $\geq 65$ years) who received the SOC intervention had a 2.47 -fold significantly higher likelihood of resuming total oral intake (aHR 2.47, 95\% CI 1.31-4.67) than their younger counterparts (50 to 64 years).

\section{Salivary flow over 14 days following extubation}

Before the intervention, $37.4 \%$ of participants met the criterion for hyposalivation (salivary flow $\leq 3 \mathrm{~cm} / 5 \mathrm{~min}$ ) 2 days postextubation. GEE analysis revealed that the SOC group had greater salivary flow over 14 days following extubation ( $\beta=0.67,95 \%$ CI $0.29-1.06, P<0.01)$ than controls. Figure 2 shows the mean salivary flow for the SOC and control groups 2, 7, and 14 days after extubation.

\section{Intervention feasibility}

Participants and their caregivers positively perceived the SOC intervention, which took on average $15.4 \mathrm{~min}$ to complete daily. Participants received on average $8.3 \pm$ 4.2 days of the SOC (range 1-14), with no reported adverse events (coughing, wet voice, or decreased oxygen saturation) during and immediately after the intervention. Adherence to SOC components was moderately good; for the sessions that participants could receive (maximum 14), adherence rates were $95.3 \%$ for 
Table 2 Time to resume total oral intake by group

\begin{tabular}{llll}
\hline Participants & SOC $(n=54)$ & Control $(n=117)^{\text {Median time, days }^{\mathrm{a}}}$ & $\begin{array}{l}\text { Adjusted hazard ratio }^{\mathrm{b}} \\
\text { [95\% confidence interval] }\end{array}$ \\
\hline All participants & 14 & 16 & $1.77^{*}[1.08,2.91]$ \\
Age $\geq 65$ years $(n=36 / 74)$ & 14 & 21 & $2.47^{*}[1.31,4.67]$ \\
Age $<65$ years $(n=18 / 43)$ & 9 & 12 & $1.32[0.55,3.16]$ \\
\hline
\end{tabular}

${ }^{\mathrm{a}}$ Median time to reach FOIS level 6, based on the Kaplan-Meier analysis

${ }^{\mathrm{b}}$ Adjusted for age, baseline intake level (FOIS level 1; levels 2-3; levels 4-7), oxygen supplementation via Cox proportional hazard modeling ${ }^{*} P<0.05$

toothbrushing/salivary gland massage, $70.0 \%$ for oral motor exercise, and $80.1 \%$ for safe-swallowing education, for an overall adherence rate of $81.8 \%$. Notably, most participants could not exercise oral motor muscles against resistance due to their weakness, dyspnea, noninvasive ventilation, and/or medication issues until 3 to 4 days after extubation, resulting in a lower adherence rate of $70.0 \%$. However, before the intervention ended, approximately $80 \%$ could exercise against resistance, and among those, 10\% could only do 3 repetitions, 20\% could do 5 repetitions, and 70\% could do 10 repetitions in the oral-motor exercise protocol.

\section{Discussion}

The most important finding of this study was that our nurse-administered SOC intervention effectively increased patients' odds of resuming total oral intake and increased salivary flow 14 days following extubation after prolonged ( $\geq 48 \mathrm{~h}$ ) endotracheal intubation. This finding is important because patients who were successfully extubated after $\geq 48 \mathrm{~h}$ endotracheal intubation often complained of dry mouth and had difficulty resuming total oral intake $[14,23]$. Our SOC intervention, offered on average for 15.4 min daily over 8 days, kept patients' oral cavity moist and clean; their lips, tongue, and jaw

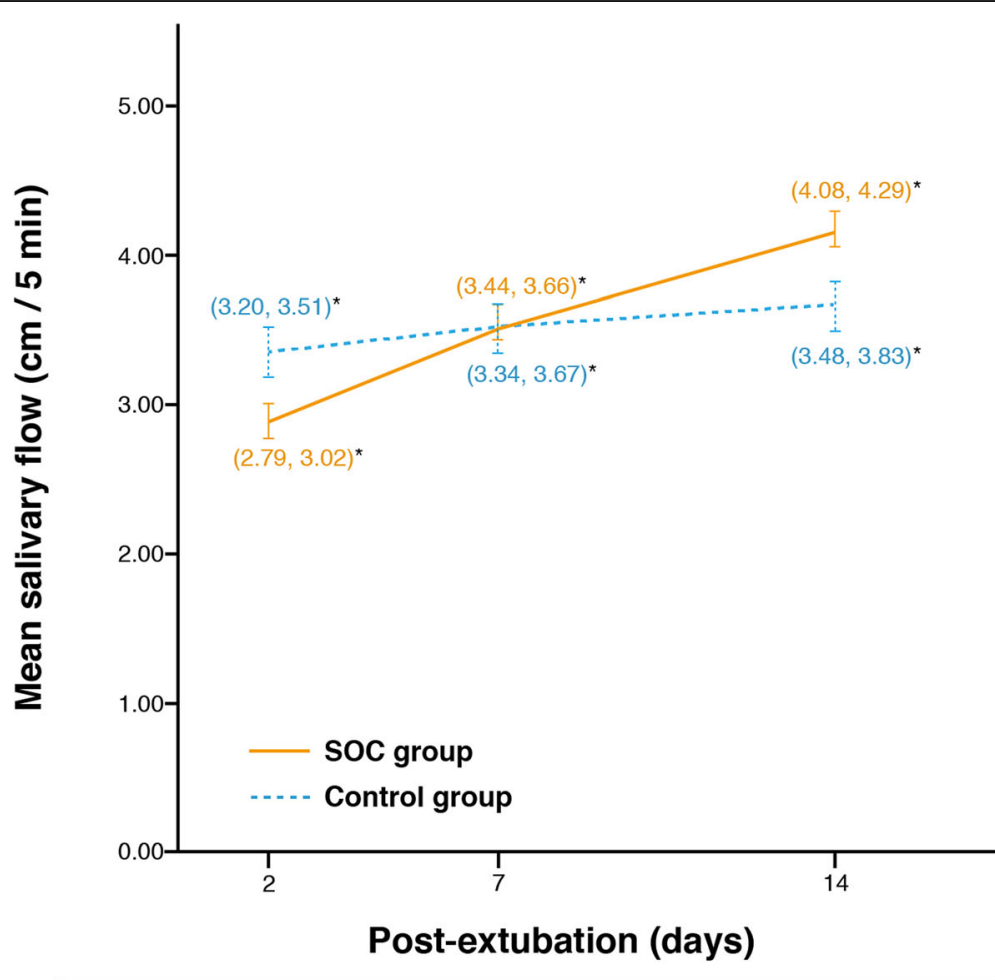

$\begin{array}{cccc}\text { Case number } & & & \\ \text { soc } & 46 & 41 & 26 \\ \text { Control } & 82 & 79 & 48\end{array}$

Fig. 2 Salivary flow 2, 7, and 14 days after extubation by group. SOC, swallowing and oral care. ${ }^{*} 95 \%$ confidence interval 
were moving freely; and patients were well-informed on safe-swallowing strategies, thus increasing their odds of resuming total oral intake 1.77 -fold.

Age mattered, with a stronger intervention effect for patients at least 65 years old; older participants who received the SOC were 2.47 -fold more likely to resume total oral intake than their younger counterparts (50 to 64 years). Moreover, salivary flow was significantly increased in patients receiving the SOC intervention following extubation. It is worth noting that participants and caregivers credited this average 8-day SOC intervention across ICU settings to the general ward as an important bridge to resuming total oral intake postextubation.

Two points warrant emphasis. First, $45.6 \%$ of participants ( $\geq 50$ years) did not resume total oral intake by 21 days after extubation, calling for effective interventions $[13,23]$. Resuming total oral intake is not spontaneous after extubation, taking 3 weeks or longer to recover, even for those with no preexisting neuromuscular disease or swallowing dysfunction. A follow-up at 3 weeks postextubation is indicated to identify patients who may benefit from referrals (e.g., rehabilitation or ear-nose-throat services) to enhance the return of oral intake function. Moreover, as a secondary prevention, the SOC intervention helps ICU patients following endotracheal extubation to resume total oral intake, especially for those $\geq 65$ years old. Future randomized controlled studies should verify our findings, understand the factors that may magnify or attenuate the SOC intervention effects, and define the SOC intervention effects in various ICU populations.

Second, targeting oral lubrication and oral sensation appears beneficial as reduced salivary flow was common among patients following endotracheal extubation, and $37.4 \%$ of our participants met the criteria for hyposalivation 2 days postextubation. This finding is consistent with a report that salivary flow was nearly absent in 24 intubated patients during their ICU stay [24]. Hyposalivation may be caused by lack of oral intake, systematic volume disturbances, medication, and stress with underlying disease. Saliva is also not distributed through the oral cavity in a supine position [25]. Indeed, oral lubrication (i.e., endogenous saliva or exogenously administered oral care products or food particles lubricate tooth-tooth, tongue-palate, and tonguemucosa interfaces) plays a vital role in effective functioning of swallowing, chewing, and tactile perception [26].

Moreover, oral sensation is important as the oral cavity has a rich somatosensory innervation and stimulating these sensory receptors in the tongue and parts of the oral cavity may improve proprioception and oral sensorimotor control in swallowing [27]. Our SOC intervention targeted oral lubrication and oral sensation, providing both salivary gland massage and toothbrushing to produce pressure and vibratory mechanical stimulation of sensory receptors in the tongue, periodontal ligaments (through pressure on the teeth), gingiva, and palate, modulating salivary flow rate [17] and somatosensory function $[15,28,29]$, all of which may improve oropharyngeal transit (i.e., swallowing efficiency). This pathophysiological mechanism is preliminarily supported by our positive findings on resuming total oral intake and increasing salivary flow. Further studies are needed to integrate the instrumental evaluation of swallowing to clarify the mechanism of how each SOC component works.

\section{Limitations}

Although our study tested the first intervention to successfully help ICU patients resume total oral intake following endotracheal extubation, it had important limitations. First, without instrumental evaluation of swallowing, it is difficult to determine the physiology underlying impairments, to what extent participants differed individually and by group, and how the SOC intervention changed swallow physiology and airway invasion to increase the odds of resuming total oral intake. Second, we did not randomize participants to the study groups, which had some substantial differences at baseline. Although these differences were carefully controlled in the Cox proportional hazard model, the control group might have been favored by the SOC participants being older and having a worse baseline intake status, minimizing the intervention effect. Third, the SOC was designed as a multicomponent "bundled" intervention, making it difficult to determine which component was most effective. Fourth, participants were recruited from one medical center with exclusion criteria, and only $66.3 \%$ of participants (128/193) completed all salivary flow tests ( 22 were reintubated, 10 died, 21 were discharged, and 12 dropped out). These factors might have limited the generalizability of our findings.

\section{Conclusions}

Despite many recent advances in ICU practice, management of dysphagia after extubation remains a significant challenge to healthcare providers and carries a significant weight of morbidity and mortality. Our study results show that a daily 15.4-min, nurse-administered SOC intervention comprising toothbrushing/salivary gland massage, oral motor exercise, and safe-swallowing education significantly increased the odds of resuming total oral intake for patients who had been successfully extubated after prolonged endotracheal intubation and had no preexisting neuromuscular disease or swallowing dysfunction. Age matters with the SOC intervention, which was more effective for those at least 65 years old.

\section{Supplementary information}

Supplementary information accompanies this paper at https://doi.org/10. 1186/s13054-019-2623-2.

Additional file 1. Swallowing and Oral Care (SOC) Intervention Protocols 


\section{Abbreviations}

FOIS: Functional oral intake scale; ROM: Range of motion; SOC: Swallowing and oral care

\section{Acknowledgments}

Not applicable.

\section{Author's contributions}

C-PW, Y-JX, T-GW, S-CK, D-CC, J-JL, T-YH, and CC-HC searched the literature. C-PW, T-GW, S-CK, and CC-HC collected the data. T-GW, S-CK, T-YH, and CCHC designed the study. C-PW, Y-JX, Y-CW analyzed the data. C-PW, Y-JX, SCK, and CC-HC prepared the manuscript. C-PW, Y-JX, T-GW, S-CK, D-CC, J-JL, $\mathrm{Y}-\mathrm{CW}, \mathrm{T}-\mathrm{YH}$, and CC-HC reviewed and approved the final manuscript.

\section{Funding}

This study was supported by the Taiwan Ministry of Science and Technology (grant no. NSC-101-2314-B-002-131-MY3).

\section{Availability of data and materials}

The datasets used and/or analyzed during the present study are available from the corresponding author on reasonable request.

\section{Ethics approval and consent to participate}

This study was approved by the Human Research Ethics Committee of National Taiwan University Hospital. All patients and/or their family members signed written informed consent to participate in the study.

\section{Consent for publication}

Not applicable.

\section{Competing interests}

The authors declare that they have no competing interests.

\section{Author details}

'Department of Nursing, National Taiwan University Hospital and National Taiwan University College of Medicine, 1, Jen-Ai Rd., Section 1, Taipei, Taiwan, Republic of China100. ${ }^{2}$ Department of Physical Medicine and Rehabilitation, National Taiwan University Hospital and National Taiwan University College of Medicine, Taipei, Taiwan, Republic of China. ${ }^{3}$ Department of Internal Medicine, National Taiwan University Hospital and National Taiwan University College of Medicine, 1, Jen-Ai Rd., Section 1 , Taipei, Taiwan, Republic of China100. ${ }^{4}$ Department of Geriatrics and Gerontology, National Taiwan University Hospital and National Taiwan University College of Medicine, Taipei, Taiwan, Republic of China. ${ }^{5}$ Superintendent's Office, National Taiwan University Hospital Zhu-Dong Branch, Hsinchu, Taiwan, Republic of China. ${ }^{6}$ Department of Dentistry, National Taiwan University Hospital and National Taiwan University College of Medicine, Taipei, Taiwan, Republic of China. ${ }^{7}$ Department of Statistics, Feng Chia University, Taichung, Taiwan, Republic of China. ${ }^{8}$ Department of Otolaryngology, National Taiwan University Hospital and National Taiwan University College of Medicine, Taipei, Taiwan, Republic of China.

Received: 4 June 2019 Accepted: 23 September 2019 Published online: 09 November 2019

\section{References}

1. Zuercher P, Moret CS, Dziewas R, Schefold JC. Dysphagia in the intensive care unit: epidemiology, mechanisms, and clinical management. Crit Care. 2019;23:103.

2. Schefold JC, Berger D, Zurcher P, Lensch M, Perren A, Jakob SM, et al. Dysphagia in mechanically ventilated ICU patients (DYnAMICS): a prospective observational trial. Crit Care Med. 2017:45(12):2061-9.

3. Macht M, Wimbish T, Clark BJ, Benson AB, Burnham EL, Williams A, et al. Postextubation dysphagia is persistent and associated with poor outcomes in survivors of critical illness. Crit Care. 2011;15:R231.

4. Kim MJ, Park YH, Park YS, Song YH. Associations between prolonged intubation and developing postextubation dysphagia and aspiration pneumonia in nonneurologic critically ill patients. Ann Rehabil Med. 2015;39:763-71.

5. Skoretz SA, Flowers HL, Martino R. The incidence of dysphagia following endotracheal intubation. Chest. 2010;137:665-73.

6. Tsai MH, Ku SC, Wang TG, Hsiao TY, Lee J, Chan DC, et al. Swallowing dysfunction following endotracheal intubation: age matters. Medicine (Baltimore). 2016;95:e3871.
7. Macht M, King CJ, Wimbish T, Clark BJ, Benson AB, Burnham, et al. Postextubation dysphagia is associated with longer hospitalization in survivors of critical illness with neurologic impairment. Crit Care. 2013;17:R119.

8. Brodsky MB, Huang M, Shanholtz C, Mendez-Tellez PA, Palmer JB,

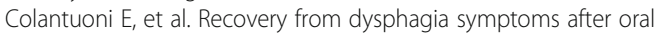
endotracheal intubation in acute respiratory distress syndrome survivors. A 5-year longitudinal study. Ann Am Thorac Soc. 2017;14:376-83.

9. Oliveira ACM, Friche AAL, Salomao MS, Bougo GC, Vicente LCC. Predictive factors for oropharyngeal dysphagia after prolonged orotracheal intubation. Braz J Otorhinolaryngol. 2018;84(6):722-8.

10. El Solh A, Okada M, Bhat A, Pietrantoni C. Swallowing disorders post orotracheal intubation in the elderly. Intensive Care Med. 2003;29:1451-5.

11. Ponfick $M$, Linden $R$, Nowak DA. Dysphagia-a common, transient symptom in critical illness polyneuropathy: a fiberoptic endoscopic evaluation of swallowing study. Crit Care Med. 2015:43(2):365-72.

12. Zielske J, Bohne S, Brunkhorst F, Axer H, Guntinas-Lichius O. Acute and long-term dysphagia in critically ill patients with severe sepsis: results of a prospective controlled observational study. Eur Arch Otorhinolaryngol. 2014;271(11):3085-93.

13. Heffner JE. Swallowing complications after endotracheal extubation: moving from "whether" to "how". Chest. 2010;137:509-10.

14. Chen CC, Wu KH, Ku SC, Chan DC, Lee JJ, Wang TG, et al. Bedside screen for oral cavity structure, salivary flow, and vocal production over the 14 days following endotracheal extubation. J Crit Care. 2018;45:1-6.

15. Su H, Hsiao TY, Ku SC, Wang TG, Lee JJ, Tzeng WC, et al. Tongue weakness and somatosensory disturbance following oral endotracheal extubation. Dysphagia. 2015:30:188-95.

16. Rassameehiran S, Klomjit S, Mankongpaisarnrung C, Rakvit A. Postextubation dysphagia. Proc (Bayl Univ Med Cent. 2015;28:18-20.

17. Affoo RH, Trottier $K$, Garrick R, Mascarenhas T, Jang Y, Martin RE. The effects of tooth brushing on whole salivary flow rate in older adults. Biomed Res Int. 2018;3904139.

18. Ono K, Uchiyama K. Evaluation of a new salivary gland massage with oil for elderly nursing home residents. Int J Phys Ther Rehabil. 2017;3:129.

19. Park T, Kim Y. Effects of tongue pressing effortful swallow in older healthy individuals. Arch Gerontol Geriatr. 2016;66:127-33.

20. Byeon $\mathrm{H}$. Effect of orofacial myofunctional exercise on the improvement of dysphagia patients' orofacial muscle strength and diadochokinetic rate. J Phys Ther Sci. 2016:28:2611-4.

21. Crary MA, Mann GD, Groher ME. Initial psychometric assessment of a functional oral intake scale for dysphagia in stroke patients. Arch Phys Med Rehabil. 2005;86:1516-20.

22. López-Jornet P, Camacho-Alonso F, Bermejo-Fenoll A. A simple test for saliva gland hypofunction using oral Schirmer's test. J Oral Pathol Med. 2006;35:244-8

23. Perren A, Zürcher P, Schefold JC. Clinical approaches to assess postextubation dysphagia (PED) in the critically ill. Dysphagia. 2019:1-12.

24. Dennesen $P$, van der Ven A, Vlasveld M, Lokker L, Ramsay G, Kessels A, et al. Inadequate salivary flow and poor oral mucosal status in intubated intensive care unit patients. Crit Care Med. 2003;31:781-6.

25. Mese H, Matsuo R. Salivary secretion, taste and hyposalivation. J Oral Rehabil. 2007;34:711-23.

26. Sarkar A, Andablo-Reyes E, Bryant M, Dowson D, Neville A. Lubrication of soft oral surfaces. Curr Opin Colloid Interface Sci. 2019;39:61-75.

27. Haggard P, deBoer L. Oral somatosensory awareness. Neurosci Biobehav Rev. 2014:47:469-84

28. Boliek CA, Rieger JM, Li SY, Mohamed Z, Kickham J, Amundsen K. Establishing a reliable protocol to measure tongue sensation. J Oral Rehabil. 2007:34:433-41.

29. Rech RS, Baumgarten A, Colvara BC, Brochier CW, de Goulart BNG, Hugo FN, et al. Association between oropharyngeal dysphagia, oral functionality, and oral sensorimotor alteration. Oral Dis. 2018;24:664-72

\section{Publisher's Note}

Springer Nature remains neutral with regard to jurisdictional claims in published maps and institutional affiliations. 